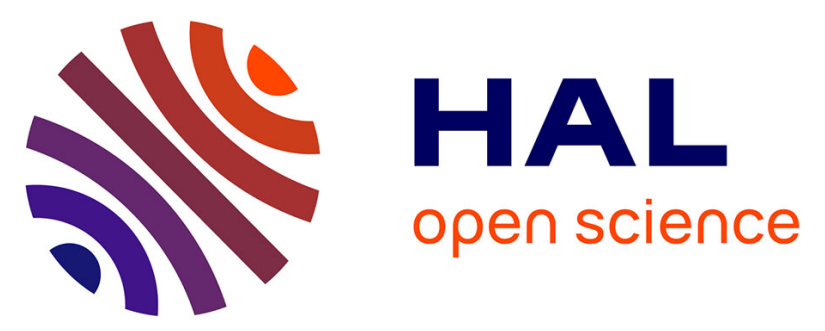

\title{
Development of liquid chromatography methods coupled to mass spectrometry for the analysis of substances with a wide variety of polarity in meconium.
}

Marie Meyer-Monath, Claudine Chatellier, Deirdre Cabooter, Florence

Rouget, Isabelle Morel, Francois Lestremau

\section{To cite this version:}

Marie Meyer-Monath, Claudine Chatellier, Deirdre Cabooter, Florence Rouget, Isabelle Morel, et al.. Development of liquid chromatography methods coupled to mass spectrometry for the analysis of substances with a wide variety of polarity in meconium.. Talanta, 2015, 138, pp.231-239. 10.1016/j.talanta.2015.02.058 . hal-01141722

HAL Id: hal-01141722

https://hal-univ-rennes1.archives-ouvertes.fr/hal-01141722

Submitted on 29 Oct 2015

HAL is a multi-disciplinary open access archive for the deposit and dissemination of scientific research documents, whether they are published or not. The documents may come from teaching and research institutions in France or abroad, or from public or private research centers.
L'archive ouverte pluridisciplinaire HAL, est destinée au dépôt et à la diffusion de documents scientifiques de niveau recherche, publiés ou non, émanant des établissements d'enseignement et de recherche français ou étrangers, des laboratoires publics ou privés. 


\section{ACCEPTED MANUSCRIPT}

Development of liquid chromatography methods coupled to mass spectrometry for the analysis of substances with a wide variety of polarity in meconium

Marie MEYER-MONATHa, Claudine CHATELLIERa, Deirdre CABOOTERc, Florence ROUGETd,e, f, Isabelle MORELb, Francois LESTREMAUa*

a INERIS, Direction des Risques Chroniques, Parc Technologique ALATA - BP 2, 60550 Verneuil-en-Halatte, France

b Laboratoire de Toxicologie Biologique et Médico-légale, CHU and INSERM 991, F-35033

Rennes, France

c KU Leuven, Department for Pharmaceutical and Pharmacological Sciences, Pharmaceutical Analysis, Herestraat 49, Leuven, Belgium

d Brittany Registry of Congenital Malformations, Rennes, France

e Department of Pediatrics, Rennes University Hospital, France

d National Institute for Health and Medical Research (Inserm) U1085, IRSET, Rennes, France

*Corresponding author. Tel.: +33 3445565 20; fax: +33 344556872

E-mail address: francois.lestremau@ineris.fr (Lestremau, F) 


\section{ACCEPTED MANUSCRIPT}

Highlights

Meconium is a fetal matrix which integrates a large exposure window to xenobiotics

Analysis of 28 target compounds with a large range of polarity

Comparison of six columns for analytical method development by LC/MS/MS in meconium

Optimization of HILIC conditions for polar compounds in meconium analysis 
Abstract

Meconium is the first fecal excretion of newborns. This complex accumulative matrix allows assessing the exposure of the fetus to xenobiotics during the last six months of pregnancy. To determine the eventual effect of fetal exposure to micropolluants in this matrix, robust and sensitive analytical methods must be developed. This article describes the method development of liquid chromatography methods coupled to triple quadrupole mass spectrometry for relevant pollutants. The 28 selected target compounds had different physico chemical properties from very polar (glyphosate) to non-polar molecules (pyrethroids). Tests were performed with six different columns: reversed phase, ion exchange and HILIC. As a unique method could not be determined for the simultaneous analysis of all compounds, three columns were selected and suitable chromatographic methods were optimized. Similar results were noticed for the separation of the target compounds dissolved in either meconium extract or solvent for reversed phase and ion exchange columns. However, for HILIC, the matrix had a significant influence on the peak shape and robustness of the method. Finally, the analytical methods were applied to "real" meconium samples.

Keywords: reversed phase; ion exchange; HILIC; LC/MS/MS; meconium; micropollutants 
Introduction

Like the general population, pregnant women are commonly exposed to many environmental pollutants (pesticides, solvents...) which have been determined harmful to humans due to their carcinogenic properties and/or their effects on reproduction. To determine the exposure to micropollutants of women during pregnancy, the PELAGIE project (Perturbateurs Endocriniens: Étude Longitudinale sur les Anomalies de la Grossesse, l'Infertilité et l'Enfance), wherein maternal urine and cord blood samples were analyzed, was carried out in Brittany, France [1]. The results of this study identified some important compounds of interest, such as organophosphorus pesticides and dialkylphosphates (metabolites of organophosphorus) [2]. The constant exposure of pregnant women to these compounds could affect the development of the fetus and especially induce the production of congenital malformations. To determine if an association exists between congenital malformations and fetal exposure to organic pollutants, a new project, the PENEW project (Pregnancy Environment and NEWborn malformations), has recently been launched by the Registry of congenital malformations in Brittany, France. This project includes the study of compounds quantified in the PELAGIE project (organophosphorus pesticides) and other relevant molecules widely used in Brittany and suspected to be toxic for the fetus such as other pesticides (triazoles, pyrethroids, glyphosate...) and VOCs; with some associated metabolites. To evaluate direct fetal exposure to xenobiotics throughout pregnancy, fetal matrix was considered. Commonly used fetal matrices, such as cord blood, urine and newborn blood, only reflect the last days of exposure. Therefore, the meconium matrix was selected to represent a larger exposure window. Meconium is the first stool of a newborn. It starts forming during the 12th-13th week of gestation in intestinal compartments and accumulates until birth $[3,4,5]$. It is a very complex matrix [6] composed of water $(\sim 70 \%)$ and lipophilic 


\section{ACCEPTED MANUSCRIPT}

compounds ( $\sim 30 \%)$ : lipids, proteins, bile acids, enzymes, lanugo... Usually, it is expelled by the newborn within 24 hours after birth. The collection of meconium is non-intrusive for the newborn and simple, unlike some other fetal matrices (urine, blood).

Meconium was already used to quantify fetal exposure to drugs and metabolites $[7,8]$, alcohol metabolites $[9,10]$ or pesticides $[11,12]$ with the analysis performed by liquid chromatography coupled with tandem mass spectrometry LC/MS/MS. Usually these analytical methods were focused only on restricted classes of compounds and eventually their metabolites $[8,9,13]$. To determine a possible link with malformations, the target compounds of the PENEW project regrouped a large variety of pollutants within several families of pesticides, their metabolites, VOCs [14] and metabolites. Compared to a previous study carried out by our group where the analysis of certain micropollutants was performed on a C18 column [12], the current list of compounds included additional pesticides (epoxiconazole, tebuconazole, 2,4-D...) and also, notably, substances with stronger polarity (glyphosate) or different polar functional groups (acid compounds with the metabolites of VOCs). As the considered molecules encompassed a much larger variety in physicochemical properties, ranging from very polar to non-polar, the previously developed method was inadequate for the extended list of substances.

Analytical method development was therefore performed for the 28 substances in meconium. Extraction methods for these compounds have been investigated and described elsewhere [15]. The aim of the method development by LC/MS/MS was to obtain one or several methods suited for all target substances with acceptable retention times (not eluted in the void volume), peak shapes and sensitivity with the fewest constraints. Analysis time was also considered due to the large number of samples to be analyzed (around 235 meconium samples). Tests on several columns with different properties, buffer optimization and matrix effects are described in this article. Chromatographic tests with meconium matrix were carried 


\section{ACCEPTED MANUSCRIPT}

out after the sample preparation optimized in this matrix [15]. The final analytical method was applied to real PENEW “cases".

Experimental

Chemicals and materials

O-cresol (o-C, purity: 99.9\%), hippuric acid (HA, 98.0\%), 2-methylhippuric acid (2-MHA, 98.0\%), 3-methylhippuric acid (3-MHA, 98.0\%), 4-methylhippuric acid (4-MHA, 98.0\%), phenylglyoxylic acid (PGA, 98.0\%), S-phenylmercapturic acid (S-PMA, 99.0\%), diethylthiophosphate (DETP, 98.0\%), 2,4-dichlorophenoxyacetic acid (2,4-D, $\geq 98.0 \%)$, diazinon (purity: 98.3\%), cypermethrin (98.5\%), cyfluthrin $(99.8 \%)$, deltamethrin $(99.7 \%)$, clopyralid (99.3\%), glyphosate $(99.2 \%)$, tebuconazole $(99.5 \%)$, propoxur (99.8\%), dichlorvos (99.9\%) and benzoic acid-d5 (BA-d5, 99.0\%) were purchased from Sigma-Aldrich Chemie GmbH (Steinheim, Germany). S-benzylmercapturic acid (S-BMA, purity: 98.0\%) was obtained from Sigma-Aldrich (Milwaukee, WI, USA). Chlorpyrifos (purity: 99.9\%), malathion $(97.2 \%)$, epoxiconazole $(99.2 \%)$ were purchased from Riedel-de Haën (Seelze, Germany). Dimethylthiophosphate (DMTP, 99.2\%), dimethyldithiophosphate (DMDTP, 99.4\%), 3-(2,2-dichlorovinyl)-2,2-dimethylcyclopropanecarboxylic acid (DCCA, purity $\geq$ 95.0\%, at $100 \mu \mathrm{g} . \mathrm{mL}-1$ in acetonitrile), diethylthiophosphate-d10 (DETP-d10, purity: 98.0\%, at $100 \mu \mathrm{g} . \mathrm{mL}-1$ in methanol) were obtained from Cambridge Isotope Laboratories Inc. (Andover, MA, USA). Dimethylphosphate (DMP, 98.0\%) was obtained from Acros Organics (Geel, Belgium). Permethrin (99.8\%) was purchased from Ultra Scientific (Rhode Island, USA). Mandelic acid (MA, 99.0\%) was obtained from ChromaDex (Irvine, CA, USA). Diethylphosphate (DEP, 99.5\%) was obtained from Chem Service Inc. (West Chester, PA, 


\section{ACCEPTED MANUSCRIPT}

USA). 3-(2,2-dibromovinyl)-2,2-dimethylcyclopropane-1-carboxylic acid (Br2CA, 99.0\%, at $10 \mu \mathrm{g} . \mathrm{mL}-1$ in methanol), diazinon-d10 (96.5\%, at $100 \mu \mathrm{g} \cdot \mathrm{mL}-1$ in acetonitrile), propoxur-d3 (99.5\%, at $100 \mu \mathrm{g} . \mathrm{mL}-1$ in acetonitrile), dichlorvos-d6 $(96.0 \%$, at $100 \mu \mathrm{g} . \mathrm{mL}-1$ in cyclohexane), trans-cypermethrin-d6 (98.5\%, at $100 \mu \mathrm{g} \cdot \mathrm{mL}-1$ in acetonitrile), chlorpyrifosd10 (98.0\%, at $100 \mu \mathrm{g} . \mathrm{mL}-1$ in acetonitrile), tebuconazole-d6 $(95.0 \%$, at $100 \mu \mathrm{g} \cdot \mathrm{mL}-1$ in acetonitrile), 3-(2,2-dichlorovinyl)-2,2-dimethylcyclopropanecarboxylic acid-d6 (DCCA-d6, $96.0 \%$, at $100 \mu \mathrm{g} . \mathrm{mL}-1$ in acetonitrile), 2,4-dichlorophenoxyacetic acid-d3 (2,4-D-d3, 97.0\%, at $100 \mu \mathrm{g} . \mathrm{mL}-1$ in acetonitrile) were purchased from Dr. Ehrenstorfer (Augsburg, Germany). Hippuric acid-d5 (HA-d5, 98.0\%) was obtained from Toronto Research Chemicals Inc. (North York, Canada).

Sodium acetate $(\mathrm{NaOAc})$, ammonium hydroxide solution $(\mathrm{NH} 4 \mathrm{OH})(99.9 \%)$, and LC-MS grade methanol $(\mathrm{MeOH})$ and acetonitrile $(\mathrm{ACN})(\geq 99.9 \%)$ were obtained from SigmaAldrich (Steinheim, Germany). Ammonium acetate $(>98 \%)$ was obtained by Merck (Darmstadt, Germany). Ultrapure water was supplied by a Mili-Q water purifier system from Millipore (Bedford, MA, USA). Acetic acid (100\%) and sodium sulfate anhydrous (NaSO4) were purchased from VWR BDH Prolabo (Leuven, Belgium).

Meconium

For method development, a pool of meconium was obtained from meconium samples collected from autopsied fetus and autopsied newborns by the anatomical pathology service of the University Hospital of Rennes, France. When tested, some of these meconium did not contain any of the analytes. These "blank" meconium were pooled to form a representative pool of "blank" meconium for use in all subsequent experiments. Meconium samples were kept at $-80^{\circ} \mathrm{C}$ until analysis to prevent bacteriological developments. 
Sample preparation

The extraction procedure of meconium specimens for quantification using the LC/MS/MS was the same as described previously [15].

Briefly, $1 \mathrm{~g}$ of meconium sample was diluted with $8 \mathrm{~mL}$ of water. Ammonium hydroxide and the internal standards were added to $6 \mathrm{~mL}$ of the diluted sample. After vortex and centrifugation, $4 \mathrm{~mL}$ of supernatant were filtered. To the remaining meconium phase, ACN, ammonium sulfate and ammonium acetate were added. The supernatant was evaporated at $500 \mu \mathrm{L}$. The two supernatants were pooled together before performing purification steps with a Bond Elut SAX cartridge (Agilent). The elutions of loading and washing steps of the Bond Elut SAX cartridge were purified with a Strata-X cartridge (Phenomenex). The elutions of the two SPE cartridges were pooled together before LC/MS/MS analysis.

Pyrethroids are not soluble in water. To avoid precipitation of pyrethroids or glyphosate in a unique solution, standard solutions were prepared in $100 \mu \mathrm{L}$ of water/ACN $(50 / 50 \mathrm{v} / \mathrm{v})$ for analysis with Ascentis Express RP-Amide and Acclaim Trinity P1 columns (one injection of $10 \mu \mathrm{L}$ for each column). For analysis with LUNA HILIC column, the $80 \mu \mathrm{L}$ of remaining solvent (mixture of $\mathrm{ACN}$ and water) in vial were evaporated to dryness and reconstituted in $80 \mu \mathrm{L}$ of ACN.

Liquid chromatography columns tests

The meconium and calibration samples were analyzed on an Acquity UPLC H-Class from Waters (Milford, MA, USA). Chromatographic development was accomplished with six columns: Ascentis Express C18, $150 \times 2.1 \mathrm{~mm}, 2.7 \mu \mathrm{m}$ from Supelco (Bellefonte, USA); 


\section{ACCEPTED MANUSCRIPT}

Ascentis Express RP-Amide, $150 \times 2.1 \mathrm{~mm}, 2.7 \mu \mathrm{m}$ from Supelco (Bellefonte, USA); Ascentis Express Phenyl-Hexyl, $150 \times 2.1 \mathrm{~mm}, 2.7 \mu \mathrm{m}$ from Supelco (Bellefonte, USA); Acclaim Trinity P1, $150 \times 2.1 \mathrm{~mm}, 3 \mu \mathrm{m}$ from Thermo Fisher Scientific (Asheville, NC, USA); Ascentis Express HILIC, $150 \times 2.1 \mathrm{~mm}, 2.7 \mu \mathrm{m}$ from Supelco (Bellefonte, USA); and LUNA HILIC, $150 \times 2.0 \mathrm{~mm}, 3 \mu \mathrm{m}$ from Phenomenex (Torrance, CA, USA).

Optimized conditions for liquid chromatography

Chromatographic separation was carried out on three columns equipped with a pre-column: Acclaim Trinity P1, Ascentis Express RP-Amide and Luna HILIC. The mobile phase consisted of ammonium acetate buffer $20 \mathrm{mM}$ set at $\mathrm{pH} 3$ with acetic acid (solvent $\mathrm{A}$ ) and acetonitrile (solvent B). The gradient program for the Ascentis Express RP-Amide column was as followed: isocratic hold at $100 \%$ A for $2 \mathrm{~min}$; from $100 \%$ A to $100 \%$ B in $8 \mathrm{~min}$; hold at $100 \% \mathrm{~B}$ for $2 \mathrm{~min}$ (total gradient time: $12 \mathrm{~min}$ ). The gradient program for the Acclaim Trinity P1 column was: isocratic hold at 95\% A for $2 \mathrm{~min}$; from 95\% A to 50\% A in 3 min (total gradient time: $5 \mathrm{~min}$ ). For the Luna HILIC column, a linear gradient from $100 \%$ B to $60 \% \mathrm{~B}$ in 5 min was used. The aqueous mobile phase was ammonium acetate buffered with acetic acid (mobile phase A) and acetonitrile as solvent (mobile phase B). The columns were constantly heated at $30^{\circ} \mathrm{C}$ for a better repeatability and the injection volume was set to $10 \mu \mathrm{L}$. All the runs were performed at a flow rate of $400 \mu \mathrm{L}$ min- 1 .

Using the current instrumentation, among different usage, the so called purge solvent is used to push the sample along the injection pathway. Therefore, the purge solvent comes into contact with the sample so its composition must be set close to the sample solvent composition. For Ascentis Express RP-Amide and Acclaim Trinity P1, the purge solvent 


\section{ACCEPTED MANUSCRIPT}

consisted of water/MeOH (90/10: v/v). For the experiments performed with the LUNA HILIC column, the purge solvent consisted of ACN alone.

Mass spectrometry

The LC was coupled to a triple quadrupole mass spectrometer Acquity UPLC TQ Detector from Waters (Milford, MA, USA) in ESI positive and negative mode. The electrospray source was used at $150^{\circ} \mathrm{C}$ with a desolvation temperature of $400^{\circ} \mathrm{C}$ and a capillary voltage of $\pm 3.30 \mathrm{kV}$ (Tables 1 and 2). Nitrogen was used as nebulizer gas with desolvation gas flow set at $800 \mathrm{~L} \mathrm{Hr}-1$, cone gas flow set at $50 \mathrm{~L} \mathrm{Hr}-1$ and argon as collision gas with flow rate set at $0.17 \mathrm{~mL}$ min-1.

For MS detection, whenever possible, two ions were used for each compound in Multiple Reaction Monitoring (MRM) mode: one target ion for quantification and one qualifier ion for confirmation (Tables 1 and 2). MassLynx software (Waters) was used for data acquisition and processing.

Results and discussion

The analysis of meconium is challenging because of the complex nature of this matrix. Each step of the analytical protocol has to be thoroughly optimized in order to reduce matrix effects. The determination of suitable chromatographic parameters is particularly critical to ensure a reliable and sensitive analysis. Separation from the matrix will ensure that interferences are reduced on peak shapes and sensitivity. Many components extracted from the matrix elute in the void volume so all target components must have a retention time of at least 2 times the void volume of the column. Moreover, even if detection by mass 


\section{ACCEPTED MANUSCRIPT}

spectrometry is selective, two transitions are used to confirm the identity of the detected components by MS/MS, this significantly decreases the possibility of obtaining a false positive by wrongly identifying a component. For compounds with adequate retention time, the selectivity provided by mass spectrometry implies that compounds that co-elute can still be identified and quantified. However, the complexity of the matrix, despite intensive sample preparation, still has a large effect on the spectrometric response as demonstrated in [15]. Therefore, the developed method must represent the best compromise between the time of analysis and the efficiency of the separation.

For this particular study, the chromatographic analysis was also a significant part of this method development because of the large polarity range of the target molecules: from very polar $(\log$ Kow $=-4.0$ for glyphosate $)$ to non-polar $(\log \mathrm{Kow}=5.7$ for deltamethrin $)$. The aim of this optimization was therefore to develop (an) easy and fast chromatographic method(s) for all target compounds.

The selection of columns was based on two criteria: adequate retention of the molecules on the column and good peak shape to maximize the sensitivity of the analysis.

Evaluation of the chromatographic separation with one column

To reduce the total analysis time, quantification of all target compounds using only one column would represent the ideal case. Considering the nature of the investigated compounds, from polar to non-polar, this column should display different types of interaction suitable for these molecules. Mixed-mode columns allow retaining different compounds with several interactions. Acclaim Trinity P1 is a trimodal column which allows different phase/molecule interactions: reversed-phase and ion exchange. These interaction modes depend on the ionization of the molecules. For chromatographic analysis, this state mainly depends on the 


\section{ACCEPTED MANUSCRIPT}

$\mathrm{pH}$ of the buffer. Tests with different $\mathrm{pH}$ were therefore performed. These experiments were carried out with $\mathrm{ACN}$ and $20 \mathrm{mM}$ buffer at $\mathrm{pH} 3$ and 6 . The flow rate was set at $400 \mu \mathrm{L}$ min-1 and the gradient program was: isocratic hold at $95 \% \mathrm{~A}$ for $2 \mathrm{~min}$; from $95 \% \mathrm{~A}$ to $95 \% \mathrm{~B}$ in $13 \mathrm{~min}$; hold at $95 \% \mathrm{~B}$ for $2 \mathrm{~min}$; then back to $95 \% \mathrm{~A}$ in $2 \mathrm{~min}$ and hold for $3.5 \mathrm{~min}$ at $95 \% \mathrm{~A}$ (total gradient time: $22.5 \mathrm{~min}$ ). Most of the target compounds were separated both at acidic and neutral $\mathrm{pH}$ (fig. 1a and 1b). For analysis at $\mathrm{pH} 3$ (fig. 1a), only two molecules could not be eluted: PGA and clopyralid while DETP was detected at 18.5 min. At pH 6 (fig. 1b), glyphosate and DMP were not or very little retained, and 2,4-D eluted at $18.9 \mathrm{~min}$. For the dialkylphosphates (DAPs), glyphosate, PGA, clopyralid and 2,4-D, the principal mechanism of retention was ion exchange. These compounds were more retained at $\mathrm{pH} 3$, except for 2,4$\mathrm{D}$ which was more retained at $\mathrm{pH}$ 6. Indeed, at $\mathrm{pH} \mathrm{3,} \mathrm{this} \mathrm{molecule} \mathrm{was} \mathrm{partially} \mathrm{ionized}$ whereas it is completely ionized at high $\mathrm{pH}$. As shown in fig. 1a and $1 \mathrm{~b}$, several compounds such as the organophosphorus pesticides, propoxur, triazoles and pyrethroids were retained through a reversed-phase mechanism as their retention was not influenced by the buffer $\mathrm{pH}$. Overall, even if most of the target compounds eluted with a retention time $\geq 2$ times the void volume, some were not retained and/or presented poor peak shapes at each $\mathrm{pH}$. This method could therefore be relevant for studies where high concentrations are expected but for this particular work aiming at traces analysis, this constituted a limitation. Therefore the present analytical method was not selected for the quantification of all target compounds in meconium. Another method using multiple columns was rather envisaged to obtain good peak shapes and retention times for all studied compounds.

Evaluation of the separation with multiple columns 
As an analysis on only one column was not possible, tests with reversed phase columns were carried out to analyze most of the target compounds. Literature showed that this type of column was suitable for pesticide analysis in different matrices, such as water [16-18] and biological matrices like urine [19] or meconium [12]. In a previous work by our group [12], a C18 column was used to analyze pesticides and their metabolites in meconium. However, this column was not suitable for this work due to the larger range of polarity and functional groups for the considered compounds. Three reversed-phase columns with different functional groups were therefore tested: Ascentis Express C18, Ascentis Express RP-Amide, and Ascentis Express Phenyl-Hexyl. Ascentis Express C18 was chosen as a reference C18 reversed phase. Ascentis Express RP-Amide was tested because it can improve the peak shape of some molecules compared to Ascentis Express C18. Finally, most of the target molecules had a phenyl group which could interact with a stationary phase containing a phenyl group such as Ascentis Express Phenyl-Hexyl.

For the tests of the 3 reversed phase columns, the same experimental conditions were used (gradient, mobile phase with buffer composed of ammonium acetate and acetic acid/ACN). The flow rate was set at $400 \mu \mathrm{L}$ min-1 and gradient program was isocratic hold at $95 \% \mathrm{~A}$ for 2 min; from $95 \%$ A to $95 \%$ B in 13 min; hold at 95\% B for 2 min; then back to $95 \%$ A in 2 min and hold for $3.5 \mathrm{~min}$ at $95 \% \mathrm{~A}$ (total gradient time: $22.5 \mathrm{~min}$ ).

The mobile phase $\mathrm{pH}$ is an important parameter for column selectivity particularly in this study given the high number of substances with ionizable groups. Therefore, buffers were tested at $\mathrm{pH} 3$ and $\mathrm{pH}$ 6. Overall, compared to the results obtained with the mixed mode Acclaim Trinity P1 column, better peak shapes were obtained for most compounds using the reversed phase columns. 


\section{ACCEPTED MANUSCRIPT}

While most compounds, such as diazinon, remained unaffected by $\mathrm{pH}$ changes, some molecules with particular functional groups such as mandelic acid or S-PMA presented distorted peaks depending on the $\mathrm{pH}$ or the column (fig. S1).

At $\mathrm{pH}$ 6, acidic compounds such as mandelic acid or S-PMA are ionized and therefore elute at or close to the void volume. Moreover, peak shapes were very broad on all columns for SPMA at $\mathrm{pH}$ 6. For this type of compound, the same behavior was noticed on the Acquity BEH $\mathrm{C} 18$ used at this $\mathrm{pH}$ in our previous work (data not shown) [12]. At $\mathrm{pH} 3$, mandelic acid and S-PMA are mainly neutral. Therefore, interactions with these stationary phases are mainly governed by hydrophobic interactions.

At $\mathrm{pH} 3$, broad peak shapes were observed for mandelic acid with Ascentis Express C18 and

for Ascentis Express Phenyl-Hexyl columns but the Ascentis RP-Amide provided an acceptable result in term of peak shapes and retention times. At this $\mathrm{pH}$, the Ascentis RPAmide appeared more suitable for the analysis of polar compounds.

The peak shape and the retention of diazinon were not influenced by $\mathrm{pH}$ because this compound is in neutral form at $\mathrm{pH} 3$ or 6.

Finally, with the 3 tested columns, glyphosate and DAPs (except DMDTP) were also not or poorly retained because of their high polarities. Therefore, the Ascentis Express RP-Amide column at pH 3 was selected (fig. 2) for the analysis of all compounds apart glyphosate and DAPs (except DMDTP).

\section{HILIC optimization}

Several published methods use C18 columns to analyze dialkylphosphates (DAPs) [20-22]. In our previous work [12], a C18 column was also used to analyze DAPs along with other pesticides. But as demonstrated, the buffer conditions which were used here $(\mathrm{pH} 6)$ were not 


\section{ACCEPTED MANUSCRIPT}

suitable for the analysis of the acidic compounds in this study. Moreover, considering the sample preparation was changed to account for the wider range of polarities of the investigated compounds in this study, interferences during the spectrometric detection were noticed with the analysis of the very polar DAPs under these conditions.

Therefore, Hydrophilic Interaction Chromatography (HILIC) was evaluated. As an alternative to normal phase, this interaction mode is used for the liquid chromatographic separation of polar and hydrophilic molecules that are not retained using reversed-phase liquid chromatography. The separation is based principally on electrostatic interactions (ionized analytes, ions contained in buffer (salt) and deprotonated silanols of stationary phase) and hydrophilic partitioning (between the buffer layer at the surface of the stationary phase and the organic layer) [23,24]. Many HILIC stationary phases are available: bare silica, polar functionalities bonded to silica or polymer coating bonded to silica.

This type of column is very sensitive to chromatographic conditions and to matrix effects. Optimization of the separation by HILIC was carried out by testing 2 different types of HILIC columns and by evaluating the nature and concentration of salt and the buffer $\mathrm{pH}$.

The two HILIC columns tested were an Ascentis Express HILIC and a LUNA HILIC. The first column contains a fused core silica phase and the second has a cross-linked diol on the silica phase.

Tests with glyphosate demonstrated that this molecule was too strongly retained on the HILIC column and could hence not be eluted. Therefore, the HILIC method optimization was focused on the analysis of DAPs.

A mixture of DAPs prepared in ACN was analyzed with the Ascentis Express HILIC column with a gradient starting at $100 \%$ ACN. Indeed, when experiments with different gradients starting with low percentage of water $(95 \%$ or $98 \%)$ were carried out, DETP was eluted in the 


\section{ACCEPTED MANUSCRIPT}

void volume of the column. With the gradient starting at $100 \% \mathrm{ACN}$, all the injections performed with the pool of meconium were reproducible in term of retention times.

An experiment was performed to check the influence of the purge solvent. The purge solvent was a mixture of water/MeOH (90/10) as used for the analysis with the reversed-phase and Acclaim Trinity P1 columns. Under these conditions, two DAPs, DETP and DMTP, eluted in the void volume of the column. Therefore, the rest of the method optimization was carried out with a mixture of standards prepared in $\mathrm{ACN}$ and with a purge solvent consisting of ACN only.

Influence of buffer

Addition of salt (compatible with MS detection) to the mobile phase allows improving retention time, selectivity and peak shapes in HILIC $[25,26]$. Indeed, charges induced by the salt create electrostatic interactions with ionized compounds [27]. To determine the influence of this parameter on the two HILIC columns, two buffers were tested: $20 \mathrm{mM}$ of ammonium acetate/acetic acid at $\mathrm{pH} 3$ and $20 \mathrm{mM}$ of ammonium formate/formic acid at $\mathrm{pH} 3$. At the same $\mathrm{pH}$, molecule retention time and peak shape depend on buffer type [26]. Better peak shapes and an increase in retention time were obtained for DEP and DMP for the Ascentis Express HILIC column with ammonium acetate as observed in other studies (fig. $3 \mathrm{a}$ and $3 \mathrm{~b}$ ). For the LUNA HILIC column, better peak shapes were also observed for DEP and DMP with this buffer (fig. 3b) while for DETP and DMTP, the two buffers provided poor retention (fig. $3 \mathrm{~b}$ and $3 \mathrm{~d}$ ). With these results, subsequent tests were carried out in ammonium acetate solution.

The effect of the salt concentration was investigated using 10, 20, 50, 80 and $100 \mathrm{mM}$ of salt in aqueous solution. On the LUNA HILIC column (fig. S2a), a slight increase of retention 


\section{ACCEPTED MANUSCRIPT}

was observed for DETP and DMTP when the salt concentration in the buffer was increased.

On the Ascentis Express HILIC column (fig. S2b), the retention times of DETP and DMTP decreased slightly with increasing ammonium acetate level. A decrease in DMP retention was observed from 10 to $50 \mathrm{mM}$ of salt which then stabilized at higher concentrations. The same phenomenon (decrease of retention time) was noticed for DETP but in the range from 10 to $20 \mathrm{mM}$. An ammonium acetate buffer concentration of $20 \mathrm{mM}$ was therefore selected.

Influence of $\mathrm{pH}$

Four $\mathrm{pH}$-values were tested: $3,4,5$ and 6.5 . The effect of mobile phase $\mathrm{pH}$ on the LUNA HILIC column is presented in fig. 4a. With $20 \mathrm{mM}$ of ammonium acetate (full markers), retention times of DETP $(\mathrm{pKa} \simeq 2.7)$ and DMTP $(\mathrm{pKa} \simeq 2.9)$ slightly increase from $\mathrm{pH} 3$ to $\mathrm{pH} 4$ but decrease for higher $\mathrm{pH}$-values to elute close to the void volume at $\mathrm{pH} 6.5$. This trend can be correlated with the $\mathrm{pKa}$ of these 2 compounds which are mainly in neutral form up to pH 4 and are then ionized for higher pHs. For DMP and DEP, a decrease was observed from pH 3 to $\mathrm{pH}$ 6.5. Indeed, DMP $(\mathrm{pKa} \simeq 2.0)$ and DEP $(\mathrm{pKa} \simeq 1.9)$ are charged negatively at $\mathrm{pH}$ 3. From $\mathrm{pH} 4$, the retention time of all compounds decreased because competition phenomena increased between ionized target molecules and increasing levels of acetate ions to create electrostatic interactions with the cross-linked diol phase. Overall, the same trend was observed with the formate buffer (empty symbols), retention times were stable from $\mathrm{pH} 3$ to $\mathrm{pH} 5$ and then decreased significantly.

On the Ascentis Express HILIC column (fig. 4b) and using ammonium acetate buffer (full symbols), no effect on the retention times of DMP and DEP was noticed when the $\mathrm{pH}$ varied between $\mathrm{pH} 3$ and $\mathrm{pH}$ 5. At $\mathrm{pH}$ values higher than 5, the ionized DEP and DMP are in competition with acetate ions (more electronegative) for electrostatic interactions accounting 


\section{ACCEPTED MANUSCRIPT}

for the decrease in retention time. For DETP/DMTP, retention times are stable over the entire $\mathrm{pH}$ range. As shown by the empty symbols, the retention times of all compounds were independent of the $\mathrm{pH}$-value when using formate buffer. The same trend as observed with the acetate buffer was again seen for DETP and DMTP. However, for DMP and DEP, no decrease in retention was observed. The electronegativity of the formate ions might be less strong than those of DEP and DMP, so their electrostatics interactions are not modified with a $\mathrm{pH}$ change.

At $\mathrm{pH}$ 6.5, several compounds were poorly retained so this $\mathrm{pH}$ was not chosen. In general, variations of retention times between $\mathrm{pH} 3,4$ and 5 were minimal. Therefore, $\mathrm{pH} 3$ was selected. With this choice, the buffer was the same as for the analysis with Ascentis Express RP-Amide.

The gradient was optimized to improve MS sensitivity, peak shapes and retention times: $100 \%$ of $\mathrm{ACN}$ to $60 \%$ at $5 \mathrm{~min}$, then $100 \%$ of $\mathrm{ACN}$ at $7 \mathrm{~min}$, hold $3 \mathrm{~min}$; with $20 \mathrm{mM}$ of ammonium acetate buffer at $\mathrm{pH} 3$ with acetic acid used. In these conditions, all compounds were retained on the Ascentis Express HILIC column (fig. 3b) but peak shapes of DMTP and DETP remained unacceptable on the LUNA HILIC column (fig. 3a).

Choice of HILIC column for meconium analysis

HILIC columns are very sensitive to matrix effects. To select a suitable column, a meconium extract containing DAPs was analyzed by LC/MS/MS. As shown in fig. 5, the chromatogram obtained for the meconium extract was very different from the chromatogram obtained in solvent (fig. 3). DMP was slightly affected by a matrix effect with good peak shape but the retention time shifted by $0.2 \mathrm{~min}$. The retention time of DEP was not modified but diffusion effects were observed as for DETP and DMTP resulting in severe band broadening. 


\section{ACCEPTED MANUSCRIPT}

Interactions between these two last compounds and meconium extract were very important.

Parts of the meconium matrix which were not removed during the purification process created interferences and interacted with the stationary phase and/or the target compounds. These interactions prevented the DAPs to link strongly with the bare silica phase.

Under identical analytical conditions, the same meconium extract was analyzed with the LUNA HILIC column (fig. 5a). In contrast to the Ascentis Express HILIC column (fig. 5b), the peak shapes were improved for DEP, DMTP and DETP compared to the analysis in solvent (fig. $3 \mathrm{~b}$ and $3 \mathrm{~d}$ ). It can be assumed that the meconium matrix had a positive effect by neutralizing the groups of the stationary phase responsible for the distorted peak shapes observed in solvent. This column was therefore selected for the analysis of the target molecules in the meconium samples.

To evaluate the importance of matrix effects further, a comparison with the chromatographic method proposed in this article and the one published by Odetokun et al. [28] in human urine was performed with the LUNA HILIC column. The chromatographic method of Odetokun et al. consisted of $93 \%$ of $\mathrm{ACN}$ and $7 \%$ of $100 \mathrm{mM}$ ammonium acetate in isocratic mode with a flow rate at $400 \mu \mathrm{L}$ min-1. In solvent, the best peak shapes were obtained for DETP and DMTP with the method of Odetokun et al. (fig. 6a) but for DEP and DMP, our optimized method provided better results. For the meconium extract (fig. 6b), our method again lead to the best peak shapes for all DAPs.

This experiment demonstrates the particular nature and effect of the meconium matrix on the chromatographic HILIC conditions. Therefore, when dealing with complex matrix such as meconium, all final optimizations should preferentially be performed in the studied matrix. 


\section{ACCEPTED MANUSCRIPT}

Usually, glyphosate is derivatized for chromatographical analysis on reversed-phase columns. For underivatized glyphosate, columns with weak anion-exchange properties can be used to obtain sufficient retention and selectivity [29,30]. Indeed, in our tests, glyphosate was not retained on the reversed-phase columns and could not be eluted with the HILIC columns. Further tests were therefore performed with the Acclaim Trinity P1 to benefit from the ion exchange interactions as seen in $\S 2.1$. Retention with the Acclaim Trinity P1 depends very much on the buffer composition: salt concentration and $\mathrm{pH}$. These two parameters were evaluated for glyphosate analysis.

Influence of $\mathrm{pH}$

Several articles on underivatizated glyphosate analysis with LC using an anion-exchange column with acidic buffer have been published [29,31]. To verify these methods, two buffer pHs were tested with $20 \mathrm{mM}$ of ammonium acetate: at pH 3 with acetic acid addition and $\mathrm{pH} 6 \mathrm{(pH}$ limit conditions indicated by the manufacturer). The flow rate was set at $400 \mu \mathrm{L}$ min- 1 and ACN was used as solvent. The gradient program was isocratic hold at $95 \%$ A for $2 \mathrm{~min}$; from $95 \%$ A to $95 \% \mathrm{~B}$ in $13 \mathrm{~min}$; hold at $95 \% \mathrm{~B}$ for $2 \mathrm{~min}$; then back to $95 \% \mathrm{~A}$ in 2 min and hold for 3.5 min at 95\% A (total gradient time: 22.5 min). At pH 6, glyphosate eluted in the void volume of the column. Indeed, at this $\mathrm{pH}$, anion exchange interactions are weak and hydrophobic interactions were insufficient to retain this compound as observed with reversed phase columns. Finally, a buffer set at $\mathrm{pH} 3$ was selected since glyphosate was retained under these conditions.

Variation of salt concentration 


\section{ACCEPTED MANUSCRIPT}

The separation optimization on a trimodal column is influenced mainly by the effect of the mobile phase ionic strength (concentration and $\mathrm{pH}$ of buffer) [32]. The influence of the buffer salt concentration on the Acclaim Trinity P1 column was tested for different concentrations of ammonium acetate $(5,10,15,20$ and $30 \mathrm{mM})$ at $\mathrm{pH} 3$. The chromatographic conditions were the same as described in the previous paragraph. As shown in fig. S3, glyphosate was sensitive to the variation of ammonium acetate concentration. Indeed, the retention time of this molecule decreased with increasing buffer concentration. With $5 \mathrm{mM}$ buffer, it was not eluted because the mobile phase had no sufficient ionic strength: interactions with glyphosate and the stationary phase were stronger than the glyphosate/mobile phase interactions. From $10 \mathrm{mM}$ of ammonium acetate on retention increased slightly $(6.42 \mathrm{~min}$ for $10 \mathrm{mM} ; 6.07 \mathrm{~min}$ for $15 \mathrm{mM}$ and $4.62 \mathrm{~min}$ for $30 \mathrm{mM}$ ) and an improvement of the peak shape was observed with a reduction of peak tailing. A buffer containing $20 \mathrm{mM}$ of ammonium acetate was therefore selected for the analysis of glyphosate on the Acclaim Trinity P1 column since it provided an acceptable result and for convenience of the overall analysis as this mobile phase was also used for the two other chromatographic methods in this work (reversed phase and HILIC).

Application to real samples

The full analytical protocol including sample preparation was validated and is described elsewhere [15]. It was applied overall to 235 meconium samples as part of the PENEW project. The meconium matrix is specific for each newborn; therefore the composition of the matrix varies for each analysis. Indeed, for the analysis of the 235 meconium samples with the LUNA HILIC column, an average retention time of 2.46 min was observed for the internal standard DETP-d10. The minimal retention time for this compound was 1.69 min and the 


\section{ACCEPTED MANUSCRIPT}

maximum 3.35 min. This deviation was not present when the matrix matched calibration was carried out with the same pool of meconium and with the quality controls inserted within the sequence of samples analysis. This phenomenon was noticed only with the analysis performed with the HILIC column demonstrating this column is particularly sensitive to matrix effects. Therefore, the use of a specific internal standard is required to take into account the variation of retention times produced by the difference in composition of the meconium samples.

Conclusion

Meconium is a complex accumulative matrix that can reflect fetal exposure to xenobiotics. To quantify 28 target compounds presenting a wide range of physico chemical properties in this matrix by LC/MS/MS, a test using only one column (of the most widely used type) did not provide satisfying results. Therefore an approach with three different complementary chromatographic methods was developed to obtain an orthogonal system suitable for all target compounds: one with a reversed-phase column for most of the target molecules (semi-polar to non-polar compounds), one with a HILIC column for polar metabolites (dialkylphosphates) and one with an ion-exchange column for a very polar pesticide (glyphosate). Analysis of meconium extracts was particularly critical using HILIC which demonstrated significant variation when analytical conditions/ matrix were changed. This analytical approach was successfully tested on 235 meconium samples.

\section{Acknowledgements}

This work was funded par the Programme Hospitalier de Recherche Clinique national (PHRC) 2010 assigned to Rennes University Hospital (CHU) for the PENEW project. It was supported by Dr Florence Rouget (Brittany Registry of Congenital Malformations) in 


\section{ACCEPTED MANUSCRIPT}

collaboration with the INSERM unities 1085 and 991 of Institut de Recherche en Santé, Environnement et Travail (IRSET) (Université Rennes I), and the Centre de Ressources Biologiques (CRB) of Centre d'Investigations Cliniques (CIC) of CHU de Rennes.

The authors thank Dr Loget of anatomical pathology service of Centre Hospitalier Universitaire (CHU) de Rennes for providing meconium samples. 
References

C. Chevrier, La cohorte Pélagie: un suivi de mères et d'enfants en Bretagne depuis 2002. Air Pur, (2010) 33-38.

C. Chevrier, C. Petit, G. Limon, C. Monfort, G. Durand, S. Cordier, Biomarqueurs urinaires d'exposition aux pesticides des femmes enceintes de la cohorte Pélagie réalisée en Bretagne, France (2002-2006), Bull Epidemiol Hebd Special Issue (2009) 23-27.

E.M. Ostrea, D.M. Bielawski, N.C. Posecion, M. Corrion, E. Villanueva-Uy, Y. Jin, J.J. Janisse, J.W. Ager, A comparison of infant hair, cord blood and meconium analysis to detect fetal exposure to environmental pesticides, Environ. Res. 106 (2008) 277-283.

D.B. Barr, A. Bishop, L.L. Needham, Concentrations of xenobiotic chemicals in the maternalfetal unit, Reprod. Toxicol. 23 (2007) 260-266.

M. Esteban, A. Castano, Non-invasive matrices in human biomonitoring: A review, Environ. Int. 35 (2009) 438-449.

C. Moore, A. Negrusz, D. Lewis, Determination of drugs of abuse in meconium, J. Chromatogr. B 713 (1998) 137-146.

T.R. Gray, D.M. Shakleya, M.A. Huestis, A liquid chromatography tandem mass spectrometry method for the simultaneous quantification of 20 drugs of abuse and metabolites in human meconium, Anal. Bioanal. Chem. 393 (2009) 1977-1990.

T.R. Gray, D.M. Shakleya, M.A. Huestis, Quantification of nicotine, cotinine, trans-3'hydroxycotinine, nornicotine and norcotinine in human meconium by liquid chromatography/tandem mass spectrometry, J. Chromatogr. B 863 (2008) 107-114.

L. Morini, E. Marchei, F. Vagnarelli, O. Garcia-Algar, A. Groppi, L. Mastrobattista, S. Pichini, Ethyl glucuronide and ethyl sulfate in meconium and hair-potential biomarkers of intrauterine exposure to ethanol, Forensic Sci. Int. 196 (2010) 74-77. 


\section{ACCEPTED MANUSCRIPT}

I. Tarcomnicu, A.L.N. van Nuijs, K. Aerts, M. De Doncker, A. Covaci, H. Neels, Ethyl glucuronide determination in meconium and hair by hydrophilic interaction liquid chromatography-tandem mass spectrometry, Forensic Sci. Int. 196 (2010) 121-127.

A.M. Tsatsakis, M.N. Tzatzarakis, D. Koutroulakis, M. Toutoudaki, S. Sifakis, Dialkyl phosphates in meconium as a biomarker of prenatal exposure to organophosphate pesticides: A study on pregnant women of rural areas in Crete, Greece, Xenobiotica 39 (2009) 364-373.

T. Berton, F. Mayhoub, K. Chardon, R. Duca, F. Lestremau, V. Bach, K. Tack, Development of an analytical strategy based on LC-MS-MS for the measurement of different classes of pesticides and theirs metabolites in meconium: Application and characterisation of foetal exposure in France, Environ. Res. 132 (2014) 311-320

E.M. Ostrea, A. Reyes, E. Villanueva-Uy, R. Pacifico, B. Benitez, E. Ramos, R.C. Bernardo, D.M. Bielawski, V. Delaney-Black, L. Chiodo, J.J. Janisse, J.W. Ager, Fetal exposure to propoxur and abnormal child neurodevelopment at 2 years of age, Neurotoxicology 33 (2012) $669-675$

M. Meyer-Monath, J. Beaumont, I. Morel, F. Rouget, K. Tack, F. Lestremau, Analysis of BTEX and chlorinated solvents in meconium by headspace-solid-phase microextraction gas chromatography coupled with mass spectometry, Anal. Bioanal. Chem. 406 (2014) 44814490.

M Meyer-Monath, C. Chatellier, F. Rouget, I. Morel, F. Lestremau, Development of a multiresidue method in fetal matrix: analysis of meconium, Anal. Bioanal. Chem. 406 (2014) 77857797

T.B. Jordan, D.S. Nichols, N.I. Kerr, Selection of SPE cartridge for automated solid-phase extraction of pesticides from water followed by liquid chromatography-tandem mass spectrometry, Anal. Bioanal. Chem. 394 (2009) 2257-2266. 


\section{ACCEPTED MANUSCRIPT}

N. Dujakovic, S. Grujic, M. Radisic, T. Vasiljevic, M. Lausevic, Determination of pesticides in surface and ground waters by liquid chromatography-electrospray-tandem mass spectrometry, Anal. Chim. Acta 678 (2010) 63-72.

N. Penner, R. Ramanathan, J. Zgoda-Pols, S. Chowdhury, Quantitative determination of hippuric and benzoic acids in urine by LC-MS/MS using surrogate standards, J. Pharmaceut. Biomed. 52 (2010) 534-543.

L. Sabatini, A. Barbieri, P. Indiveri, S. Mattioli, F.S. Violante, Validation of an HPLCMS/MS method for the simultaneous determination of phenylmercapturic acid, benzylmercapturic acid and o-methylbenzyl mercapturic acid in urine as biomarkers of exposure to benzene, toluene and xylenes, J. Chromatogr. B 863 (2008) 115-122.

F. Hernandez, J.V. Sancho, O.J. Pozo, Direct determination of alkyl phosphates in human urine by liquid chromatography/electrospray tandem mass spectrometry, Rapid Commun. Mass Sp. 16 (2002) 1766-1773.

F. Hernandez, J.V. Sancho, O.J. Pozo, An estimation of the exposure to organophosphorus pesticides through the simultaneous determination of their main metabolites in urine by liquid chromatography-tandem mass spectrometry, J. Chromatogr. B 808 (2004) 229-239.

S. Dulaurent, F. Saint-Marcoux, P. Marquet, G. Lachatre, Simultaneous determination of six dialkylphosphates in urine by liquid chromatography tandem mass spectrometry, J. Chromatogr. B 831 (2006) 223-229.

A.J. Alpert, Hydrophilic-interaction chromatography for the separation of peptides, nucleicacids and other polar compounds, J. Chromatogr. A 499 (1990) 177-196.

D. Guillarme, J.-L. Veuthey, UHPL in Life Sciences, Royal Society of Chemistry, 2012.

C. Dell'Aversano, P. Hess, M.A. Quilliam, Hydrophilic interaction liquid chromatographymass spectrometry for the analysis of paralytic shellfish poisoning (PSP) toxins, J. Chromatogr. A 1081 (2005) 190-201. 


\section{ACCEPTED MANUSCRIPT}

R.I. Chirita, C. West, A.L. Finaru, C. Elfakir, Approach to hydrophilic interaction chromatography column selection: Application to neurotransmitters analysis, J. Chromatogr. A 1217 (2010) 3091-3104.

Y. Guo, S. Gaiki, Retention and selectivity of stationary phases for hydrophilic interaction chromatography, J. Chromatogr. A 1218 (2011) 5920-5938.

M.S. Odetokun, M.A. Montesano, G. Weerasekera, R.D.Jr. Whitehead, L.L. Needham, D.B. Barr, Quantification of dialkylphosphate metabolites of organophosphorus insecticides in human urine using 96-well plate sample preparation and high-performance liquid chromatography-electrospray ionization-tandem mass spectrometry, J. Chromatogr. B 878 (2010) 2567-2574.

Y. Nagatomi, T. Yoshioka, M. Yanagisawa, A. Uyama, N. Mochizuki, Simultaneous LCMS/MS Analysis of Glyphosate, Glufosinate, and Their Metabolic Products in Beer, Barley Tea, and Their Ingredients, Biosci. Biotech. Bioch. 77 (2013) 2218-2221.

A.M. Botero-Coy, M. Ibanez, J.V. Sancho, F. Hernandez, Direct liquid chromatographytandem mass spectrometry determination of underivatized glyphosate in rice, maize and soybean, J. Chromatogr. A 1313 (2013) 157-165.

C.Y. Hao, D. Morse, F. Morra, X.M. Zhao, P. Yang, B. Nunn, Direct aqueous determination of glyphosate and related compounds by liquid chromatography/tandem mass spectrometry using reversed-phase and weak anion-exchange mixed-mode column, J. Chromatogr. A 1218 (2011) 5638-5643.

I. Birnieks, J. Maher, Acclaim Columns and Bio Columns, Dionex Corporation, CA (2009) List of figures

Fig. 1 Acclaim Trinity $\mathrm{P} 1$ chromatogram in solvent : a) at pH3 and b) at pH6: 1, PGA; 2, clopyralid; 3, MA; 4, o-cresol; 5, HA; 6, MHA; 7, S-PMA; 8, S-BMA; 9, dichlorvos; 10, 


\section{ACCEPTED MANUSCRIPT}

propoxur; 11, 2,4-D; 12, DCCA; 13, epoxiconazole; 14, malathion; 15, DMDTP; 16, Br2CA; 17, tebuconazole; 18, diazinon; 19, cyfluthrine; 20, chlorpyrifos; 21, cypermethrin; 22, deltamethrin; 23, permethrin; 24, glyphosate; 25, DMP; 26, DEP; 27, DMTP; 28, DETP

Fig. 2 Analysis of meconium spiked with target compounds by Ascentis Express RP-Amide Fig. 3 Chromatogram of DAPs in solvent analyzed with $20 \mathrm{mM}$ of ammonium acetate at $\mathrm{pH} 3$ by a) Ascentis Express HILIC and by b) LUNA HILIC; and with $20 \mathrm{mM}$ of ammonium formate at $\mathrm{pH} 3$ by c) Ascentis Express HILIC and by d) LUNA HILIC

Fig. 4 The effect of buffer $\mathrm{pH}$ on a) HILIC LUNA and b) Ascentis Express HILIC columns on the retention of DAPs. With ammonium acetate: ( ) DEP, ( ) DMP, ( ) DETP and ( ) DMTP; and with ammonium formate: ( ) DEP, ( ) DMP, ( ) DETP and ( ) DMTP Fig. 5 Chromatogram of spiked meconium analyzed with $20 \mathrm{mM}$ of buffer at $\mathrm{pH} 3$, with a) LUNA HILIC, b) Ascentis Express HILIC

Fig. 6 Chromatogram of DAPs with two chromatographic methods: Odetokun [28] conditions (dotted lines) and optimized conditions (solid lines) in solvent (a) and in spiked meconium (b) 


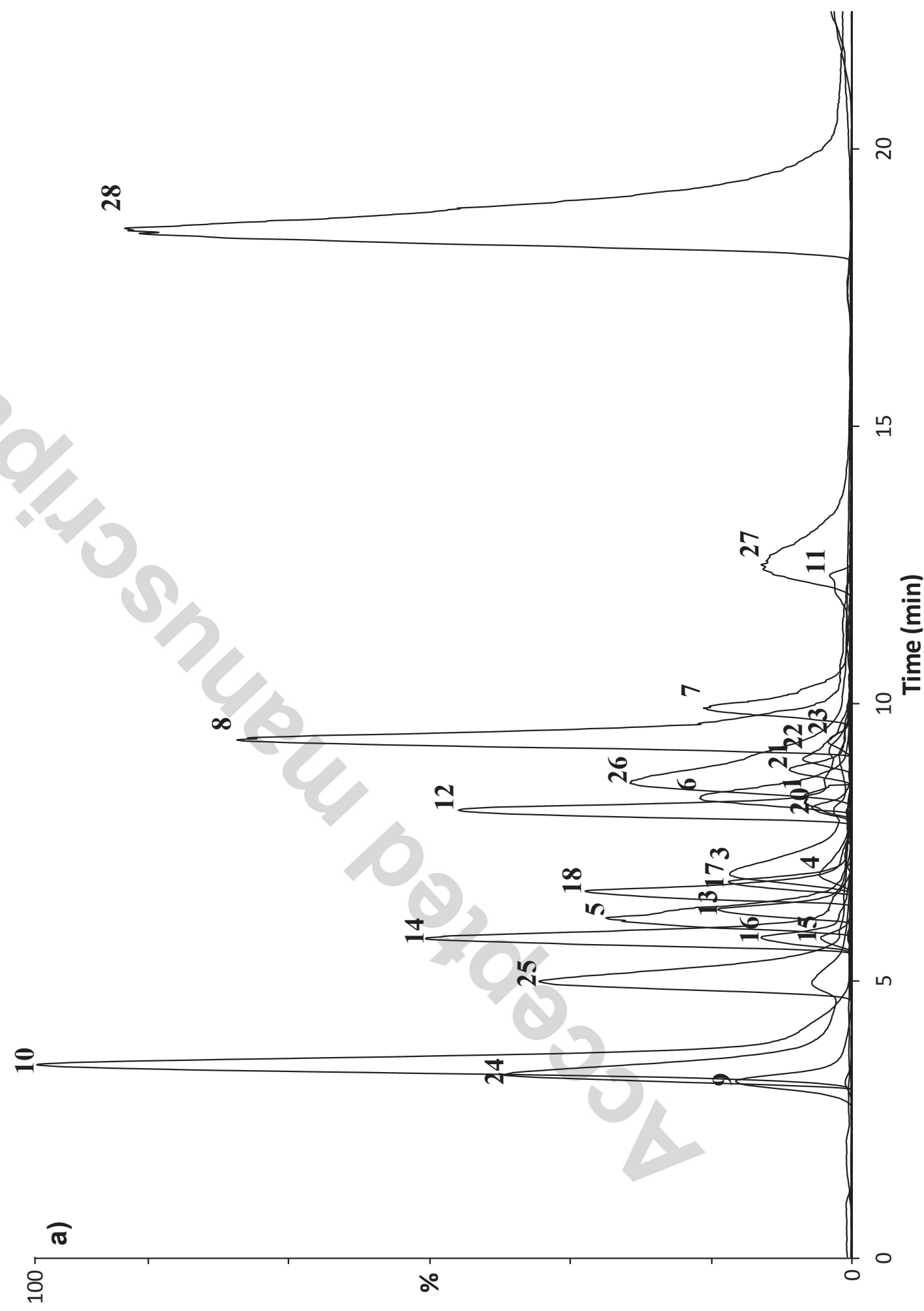




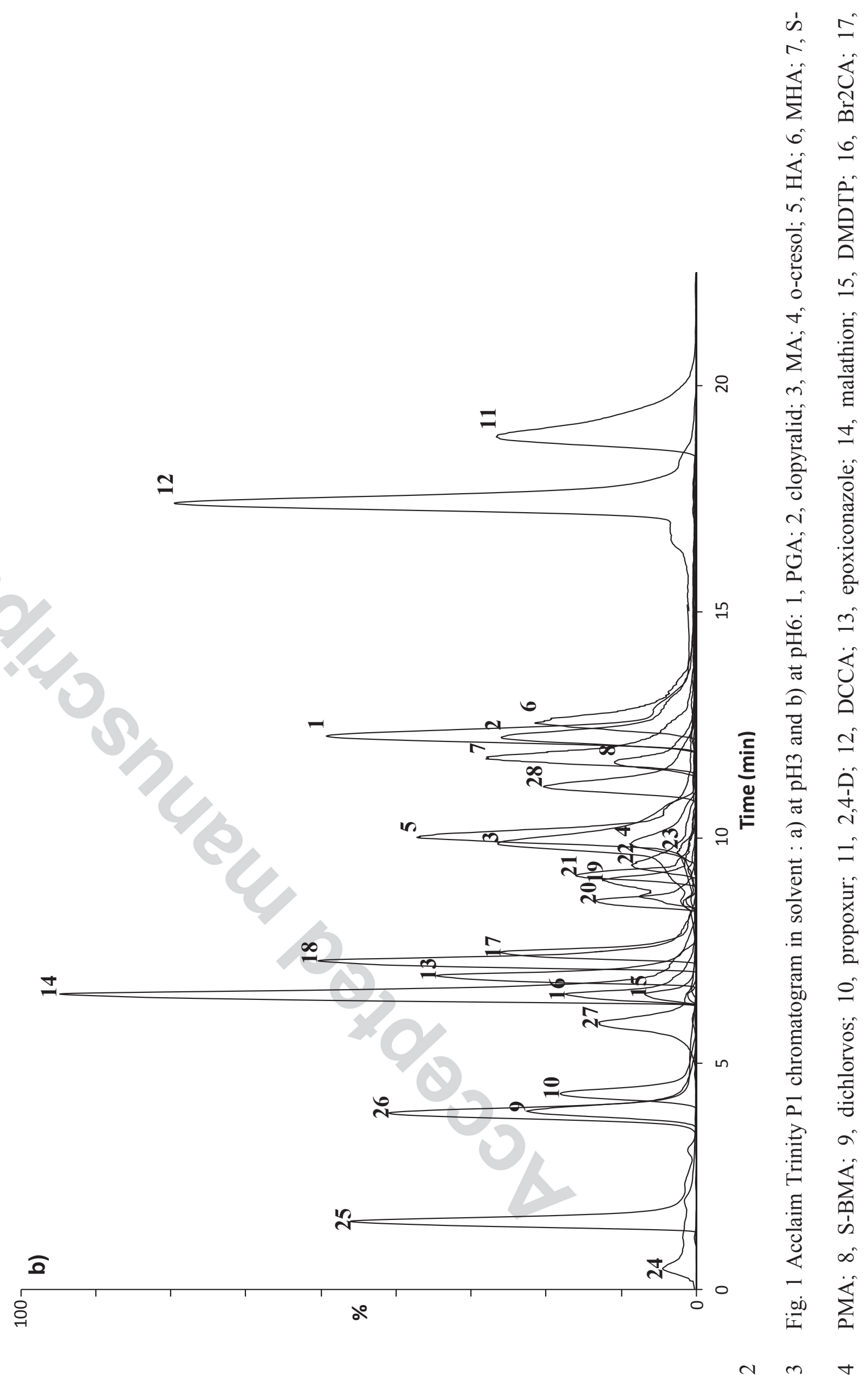




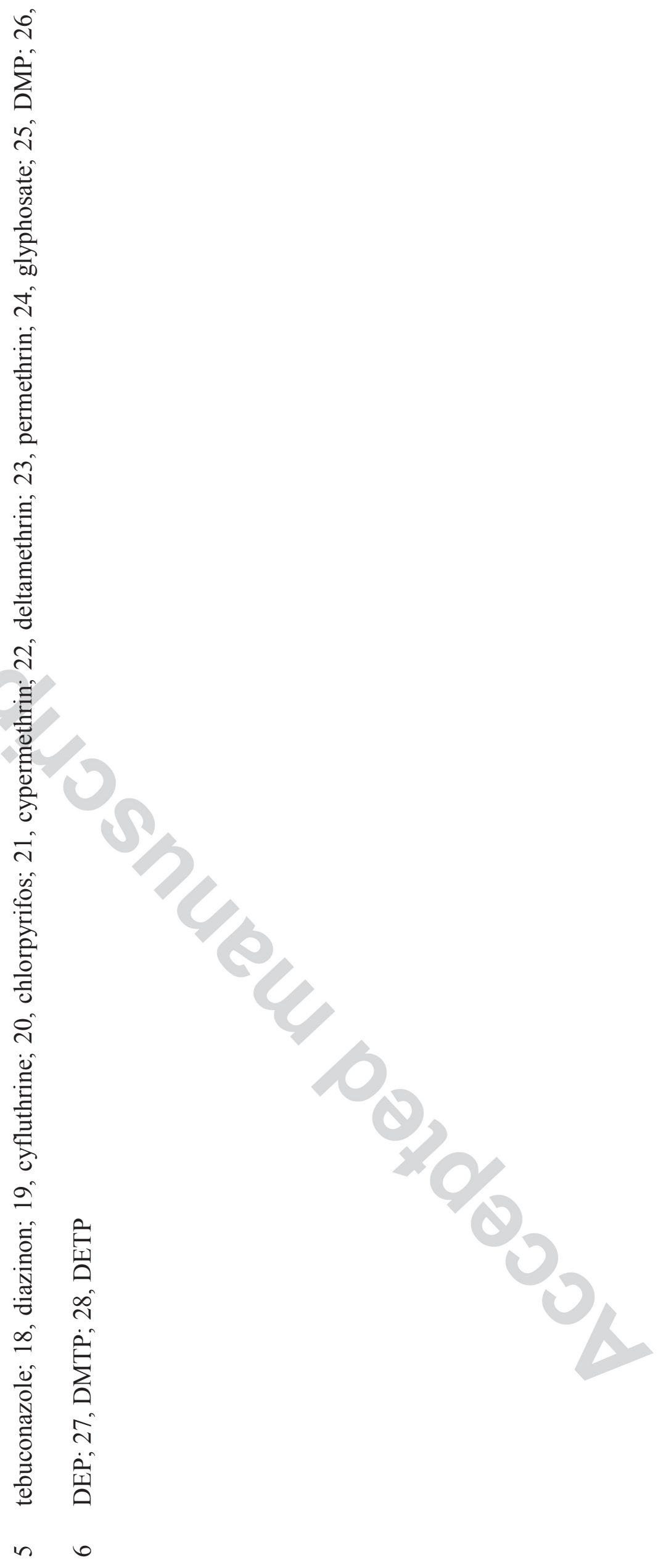




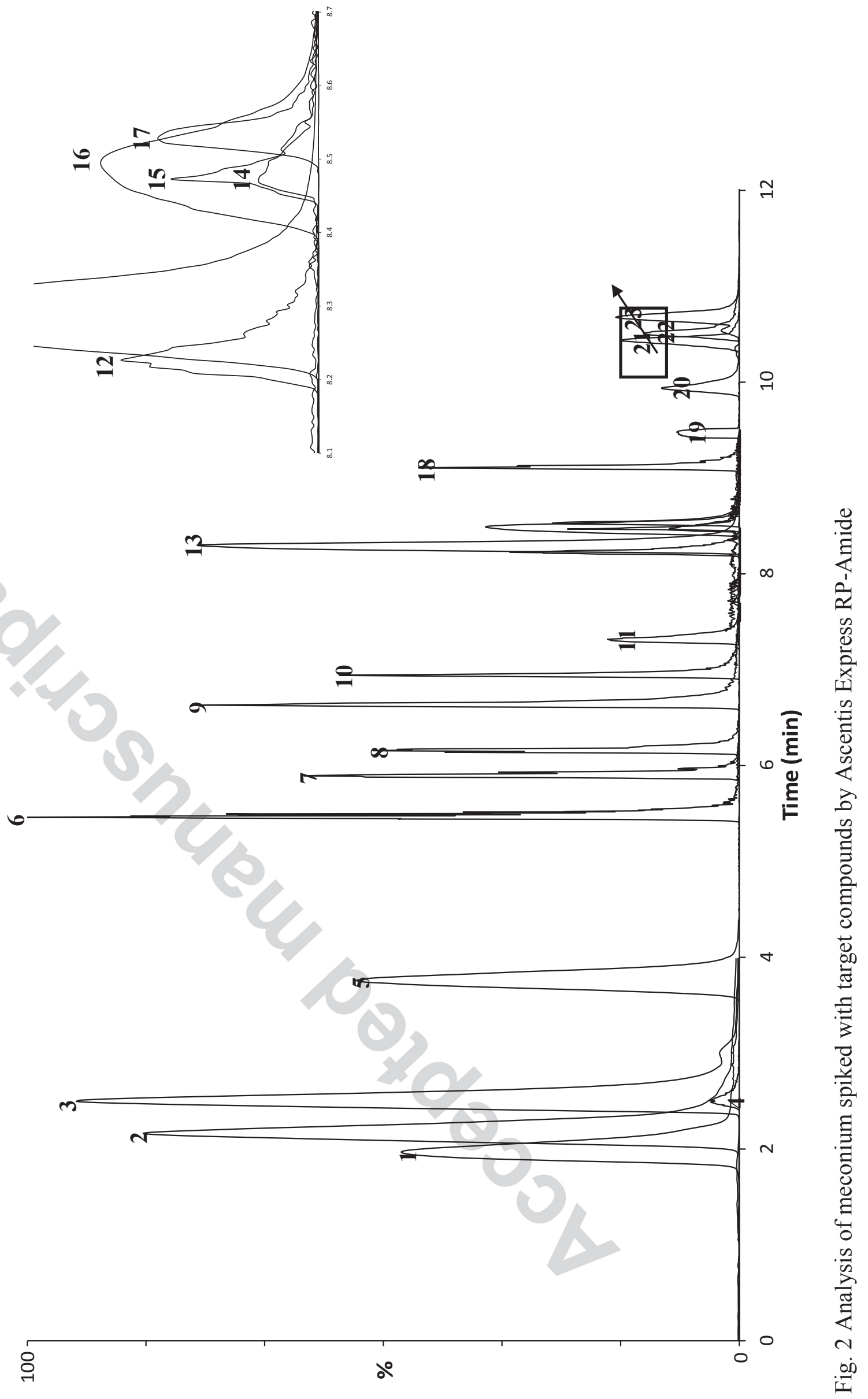



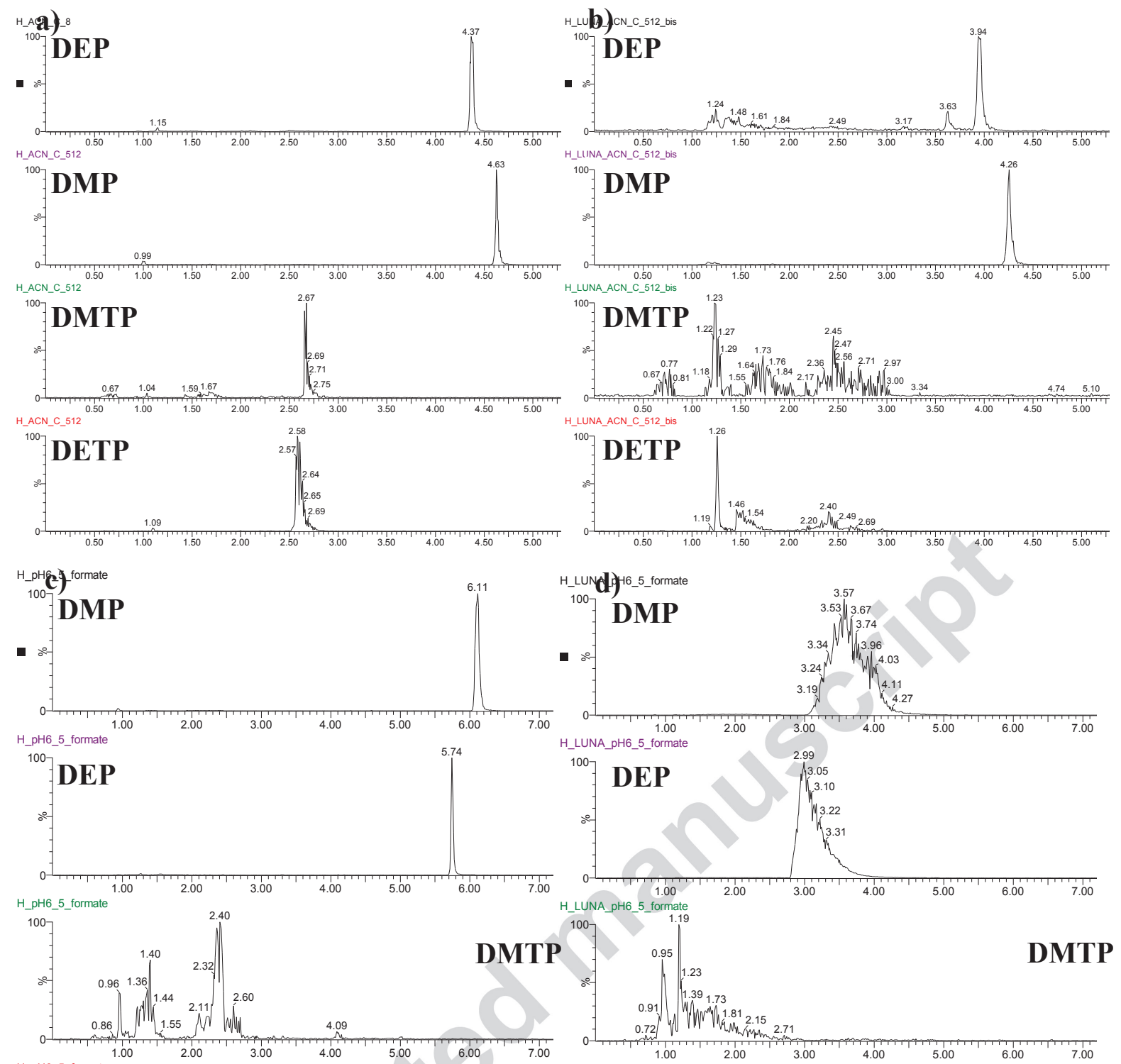

1.19
100 LUNA_PH6_5 formate
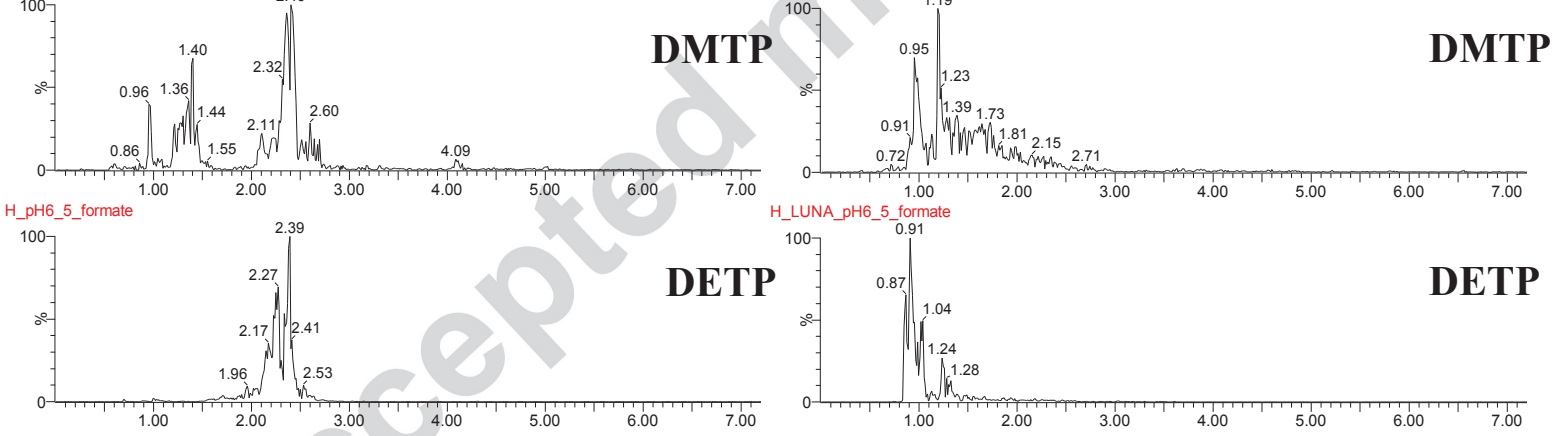

Fig. 3 Chromatogram of DAPs in solvent analyzed with $20 \mathrm{mM}$ of ammonium acetate at $\mathrm{pH} 3$ by a) Ascentis Express HILIC and by b) LUNA HILIC; and with $20 \mathrm{mM}$ of ammonium formate at $\mathrm{pH} 3$ by c) Ascentis Express HILIC and by d) LUNA HILIC 


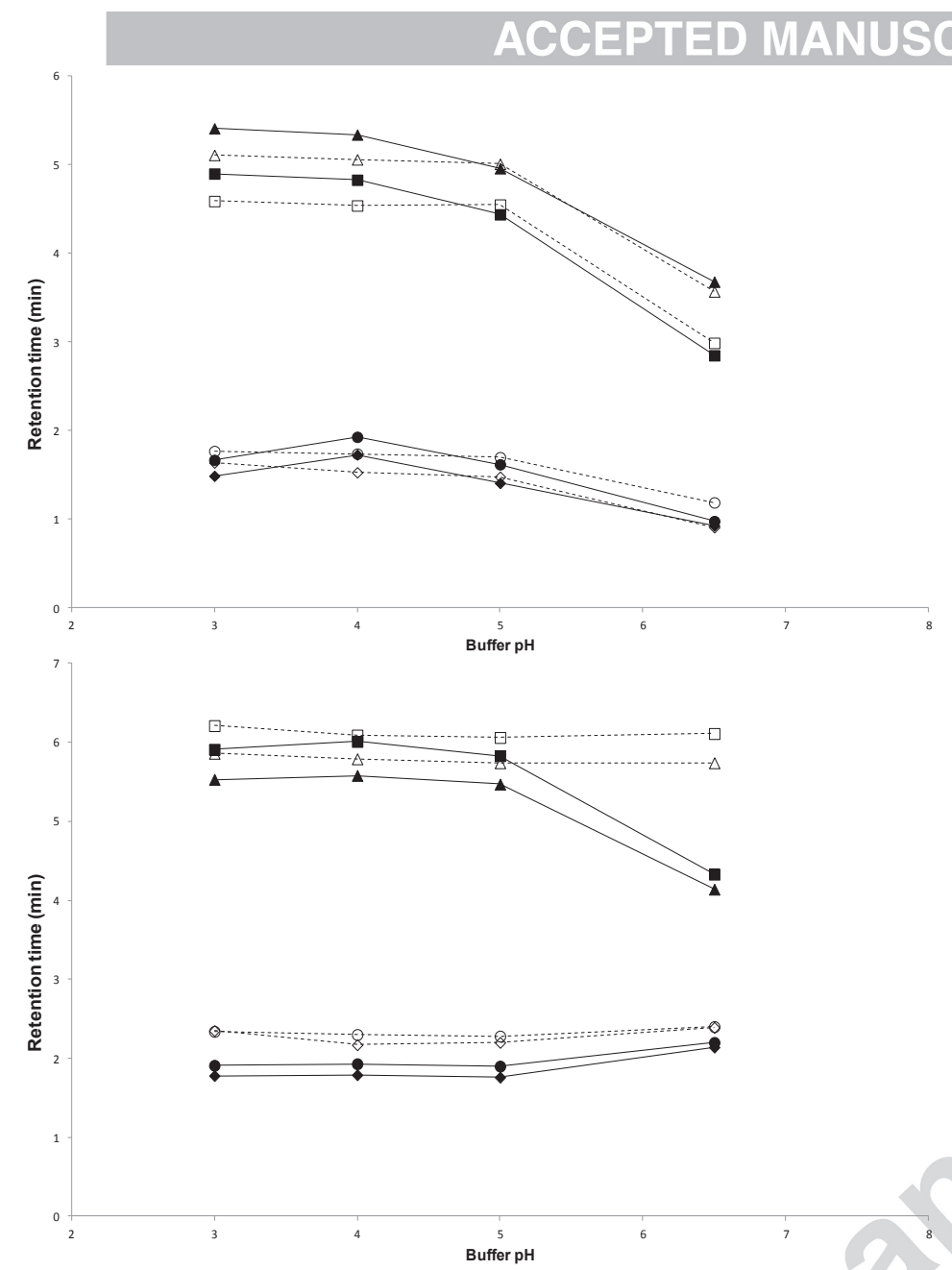

Fig. 4 The effect of buffer $\mathrm{pH}$ on a) HILIC LUNA and b) Ascentis Express HILIC columns on the retention of DAPs. With ammonium acetate: $(\boldsymbol{\square})$ DEP, $(\boldsymbol{\Delta})$ DMP, $(\boldsymbol{\nabla})$ DETP and $(\bullet)$ DMTP; and with ammonium formate: $(\square)$ DEP, $(\triangle)$ DMP, $(\diamond)$ DETP and $(O)$ DMTP 


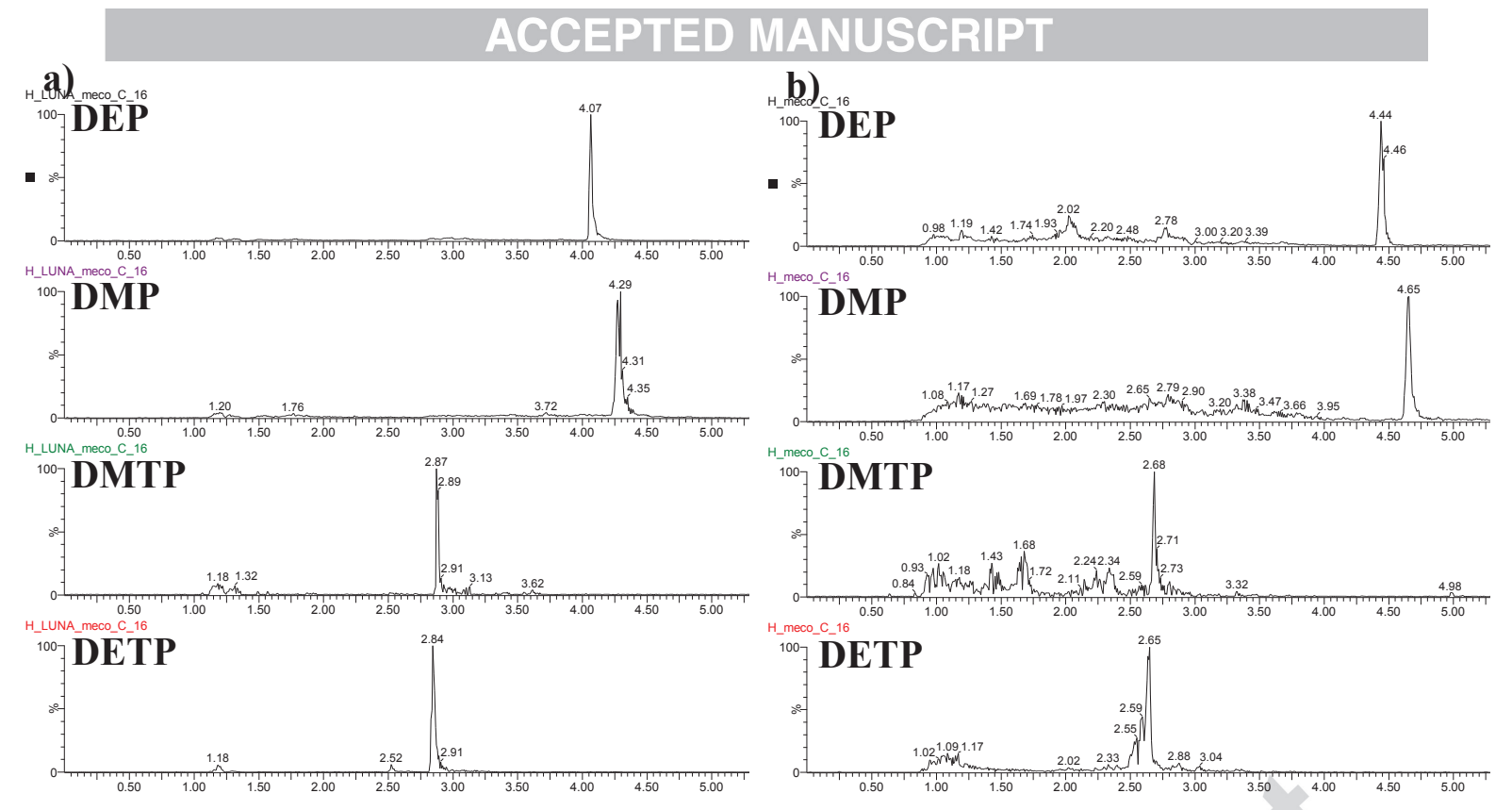

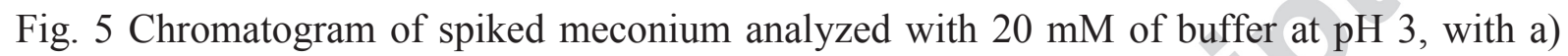
LUNA HILIC, b) Ascentis Express HILIC 

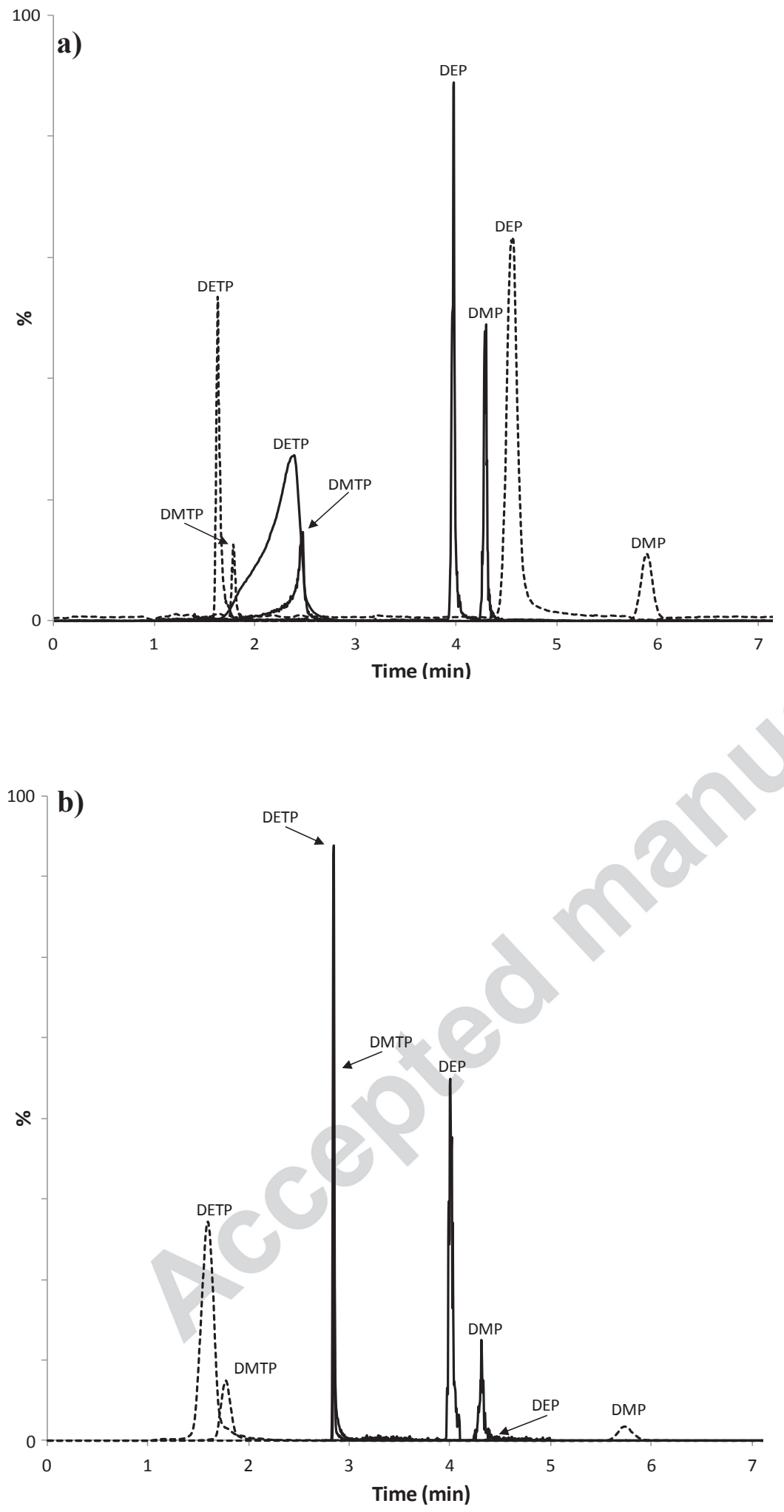

Fig. 6 Chromatogram of DAPs with two chromatographic methods: Odetokun [28] conditions (dotted lines) and optimized conditions (solid lines) in solvent (a) and in spiked meconium (b) Table 1 Chromatographic and MS parameters for target compounds 


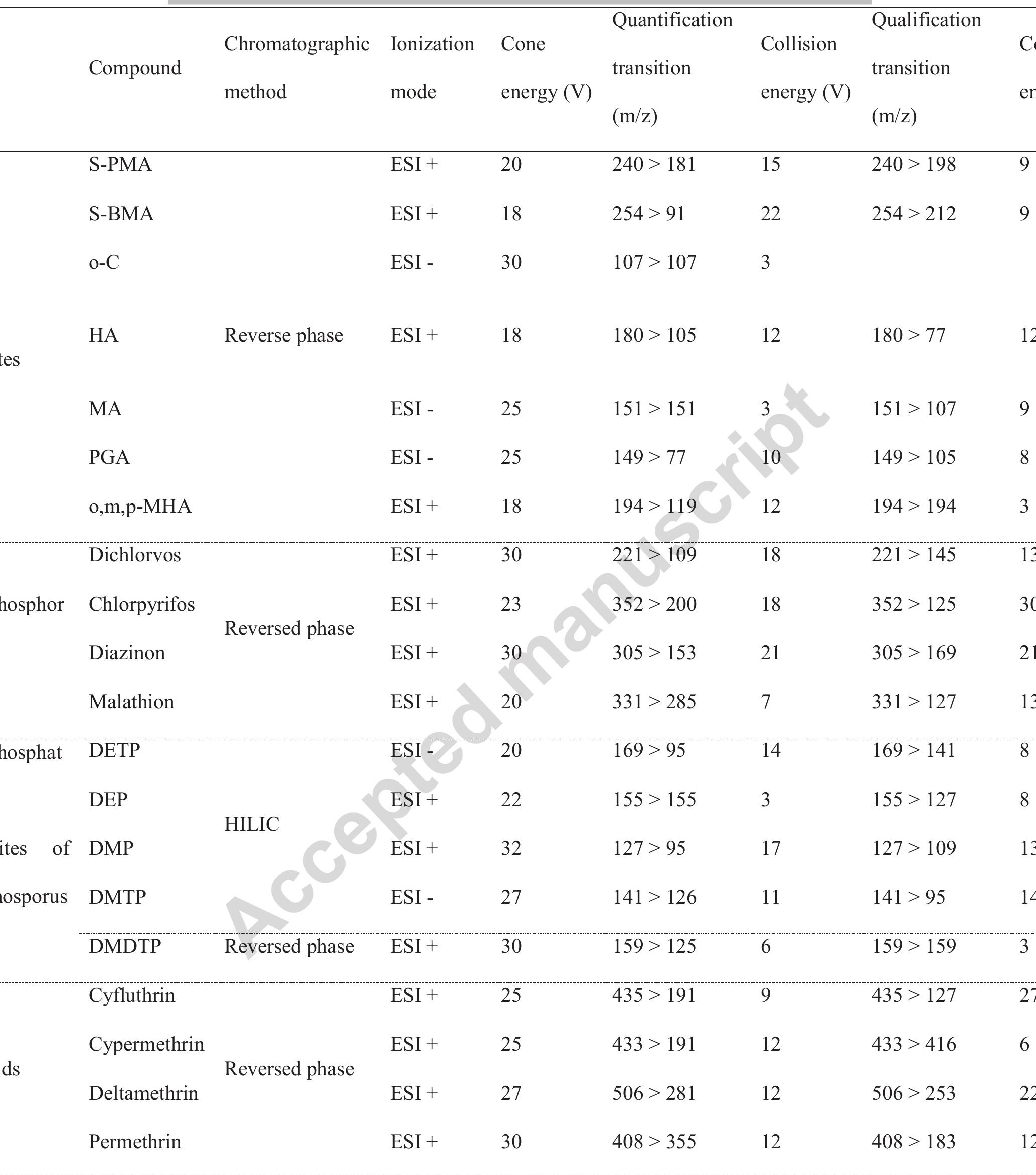




\section{ACCEPTED MANUSCRIPT}

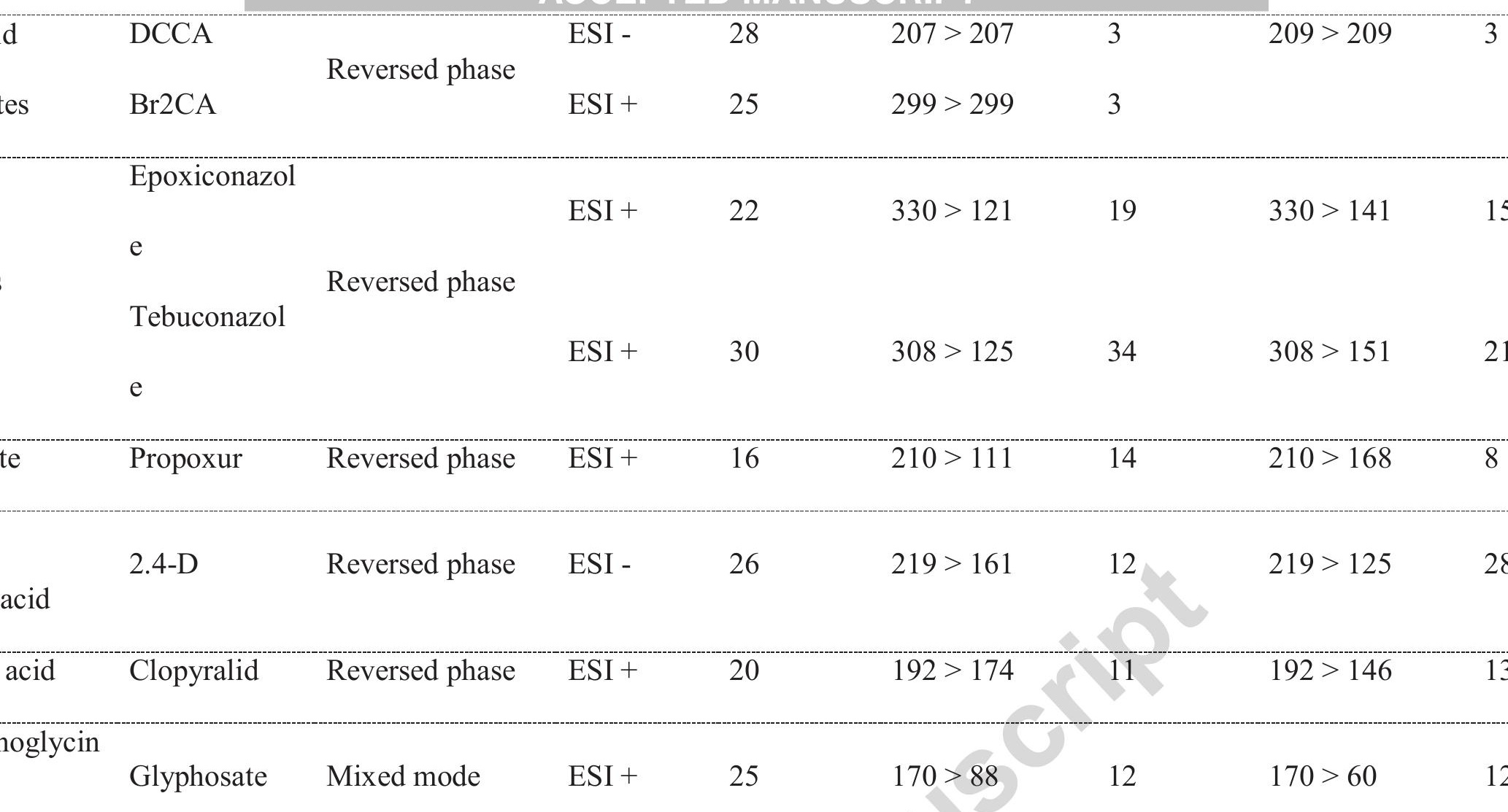

Graphical abstract

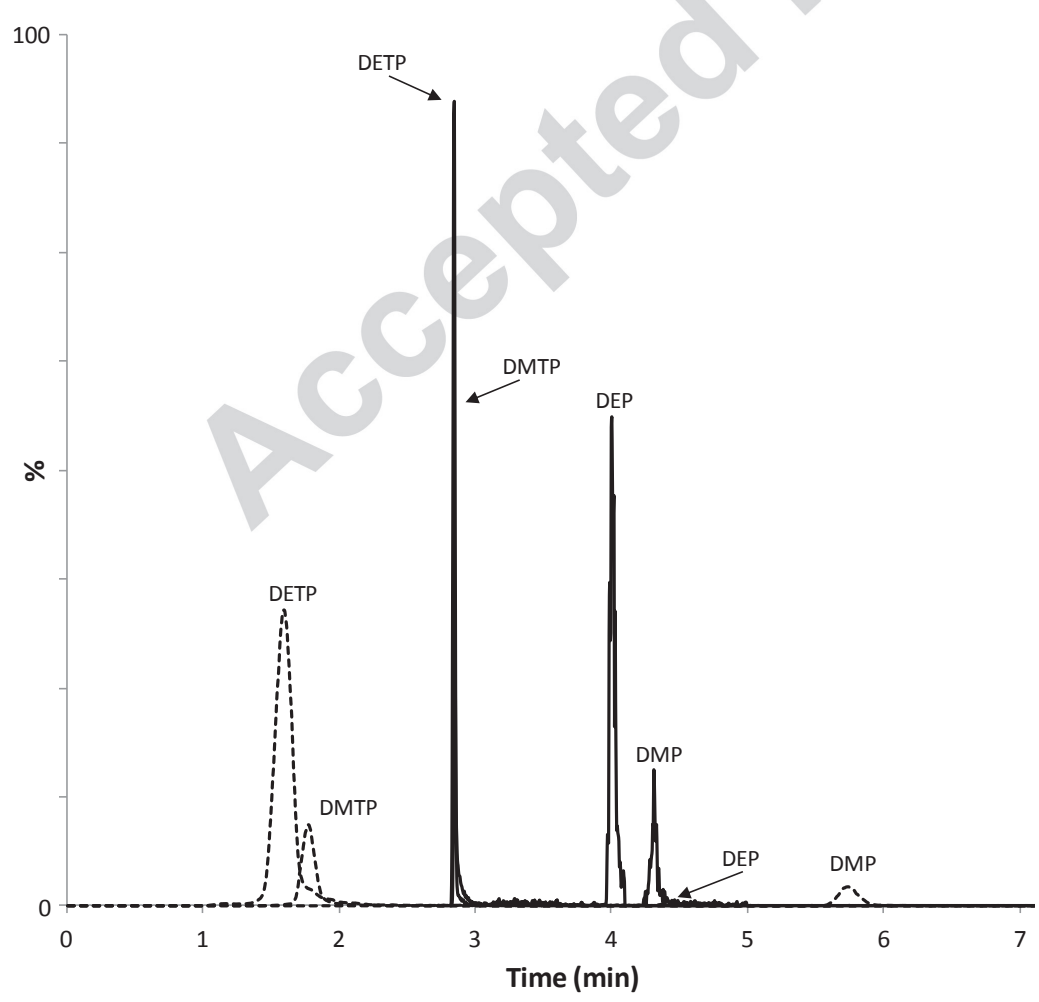




\section{ACCEPTED MANUSCRIPT}

Highlights

Meconium is a fetal matrix which integrates a large exposure window to xenobiotics

Analysis of 28 target compounds with a large range of polarity

Comparison of six columns for analytical method development by LC/MS/MS in meconium

Optimization of HILIC conditions for polar compounds in meconium analysis 